\title{
Seventeenth-Century Mechanism: An Alternative Framework for Reductionism
}

Kari L. Theurer (Trinity College)

ABSTRACT: The consensus view in philosophy of science is that reductionism is dead. One reason for this is that the deductive nomological (DN) model of explanation, on which classical reductionism depends, is widely regarded as indefensible. I argue that the DN model is inessential to the reductionist framework, and that mechanism provides a better framework for thinking about reductionism. But this runs counter to the contemporary mechanists' claim that their view provides a distinct alternative to reductionism. I demonstrate that this view is mistaken. Mechanists are committed to reductionism, as evidenced by the historical roots of the contemporary mechanist program: namely, in the mechanical philosophy of Descartes, Boyle, and others. This view shares certain core commitments with classical and contemporary reductionists. I argue that it is these shared commitments, not a direct commitment to the DN model, that constitute the essential elements of the reductionist program. 


\section{INTRODUCTION}

Among philosophers of science, the consensus view is that explanatory reductionism is dead. This is especially true in the philosophy of the biological sciences, where attempts to reduce psychology to neuroscience (e.g., Bickle 1998, 2003, 2006; Churchland 1986) and evolutionary biology to classical or molecular genetics (e.g., Rosenberg 1997, 2006) have been met primarily with scorn. This response is understandable because the classical model of reduction is indefensible. I claim that the most serious threat to the classical model stems from its dependence on the deductive nomological (DN) model of explanation, which philosophers of science have rejected almost universally. But it does not follow from the failure of the DN model that all forms of explanatory reductionism are implausible. Here, I argue that DN explanation is not an essential piece of the reductionist program, and that mechanism actually provides a better framework for thinking about reductionism than does the DN model. This claim runs counter to what many reductionists and mechanists alike suggest about their own work. Mechanists argue that their view provides a distinct alternative to reductionism. But a careful examination of the mechanical philosophy of the seventeenth century suggests that reductionism and mechanism are more tightly linked than is typically supposed. This view shares certain core commitments with classical and contemporary reductionists. I argue that it is these shared commitments, not a direct commitment to the DN model, that constitute the essential elements of the reductionist program. This close relationship between mechanism and reduction provides the groundwork for a dramatically different - and much more plausible - conception of reduction.

\section{Reduction: The Current State of Things}

The current anti-reductionist consensus can be attributed to several serious objections. Putnam (1975a) and Fodor (1974) argued forcefully that higher-level phenomena are multiply realizable, thus 
blocking the identification of higher- and lower-level properties taken to be required by classical reduction models. Others have argued that reductionism does not properly reflect the nature of scientific practice. Craver captures this worry quite succinctly:

...real explanations in neuroscience look nothing like the explanations that the reduction model requires. (Craver 2007, 109)

Scientists in most fields simply do not provide explanations that fit the reductionist model. Bickle (1998) refers to this challenge as the "put up or shut up" challenge: where are the reductions?

Much ink has been spilled in defense of reductionism against these objections in recent decades. I focus instead on a problem that few reductionists have attempted to overcome. Classical reduction, as Nagel and others envisioned it, depends essentially on the DN model of explanation (e.g., Nagel 1961, Schaffner 1967). The problem is that the DN model, which served as the gold standard of scientific explanation at the time of Nagel's writing, is now widely regarded as indefensible. Since reduction is presumed to be a species of DN explanation, it appears that reductionism is also untenable. Further exacerbating the problem is that most reductionists writing since Nagel have accepted the terms of this debate: they agree that reduction proceeds via deduction and they attempt to circumvent the resulting difficulties. My strategy is different: I grant that the problems with DN explanation are insurmountable. But I reject the presupposition that reduction depends essentially on the DN model. I conclude that mechanistic explanation provides a better framework for reductionism.

\section{An Alternative Model of Explanation: Mechanism}

Within the last decade or so, mechanism has emerged as the new standard for scientific explanation. Mechanistic explanations are causal explanations. What explains are the causal relationships between phenomena, rather than the deductive relations between sentences describing them. One prominent 
account of mechanism was initially developed by Machamer, Darden, and Craver (2000, hereafter MDC), and later expanded and refined by Craver (2007). I focus on this account, which I will call New Mechanism, because it has gained widest acceptance in the literature. I shall take it to be representative. $^{1}$

According to MDC, to give a description of a mechanism is to provide an explanation. But what is a mechanism? On their view, mechanisms "are entities and activities organized such that they are productive of regular changes from start or set-up to finish or termination conditions" (2000, 3). Explaining a phenomenon mechanistically requires breaking the system into its parts and identifying the entities and activities that contribute to the phenomenon.

Already, a few caveats are in order: neither MDC, Craver, Bechtel, nor I hold that all explanations are mechanistic explanations, or that all scientists are or should be engaged in a search for mechanisms. But I share with them the view that mechanism is an important mode of scientific explanation in many disciplines, particularly in neuroscience.

\section{Mechanism, Reduction, AND Decomposition}

The relationship between reduction and mechanism is a highly vexed one. Nagel's (1961) "classical" model of reduction requires that the laws of the reduced theory be derivable from the reducing theory plus the relevant bridge laws. Most accounts of mechanism avoid appeal to laws, and thereby cannot satisfy Nagel's conditions on reduction. For some, this apparent avoidance of reduction is central to mechanism's appeal. Mechanistic explanations do not appeal to the deductive relationships between laws that classical reduction requires. Deductive relations hold between sentences or

\footnotetext{
${ }^{1}$ For alternative conceptions of mechanism, see Bechtel and Abrahamsen 2005; Glennan 1996, 2002; Woodward 2003. While some mechanists (Glennan 1996, 2002; Schaffner 1993; Woodward 2003) appeal to laws or to lawlike generalizations, this is regarded by many mechanists unnecessary for (or even antithetical to) mechanistic explanation (Bechtel and Abrahamsen 2005; Craver 2007; MDC 2000).
} 
propositions. But mechanisms are things in the world, and as such cannot be deduced from one another.

Clearly, the New Mechanists avoid classical reduction. But this is only one of many possible formulations of the reductionist thesis. Classical reduction is a species of explanatory reduction: the more general view that higher-level phenomena can be explained in lower-level terms. ${ }^{2}$ In order to show how levels of mechanisms can be reduced, I put an old view to new use. Specifically, I appeal to the motivating insight behind Kemeny and Oppenheim's (1956) view of reduction. Their model has historically been dismissed as being too weak to support actual reductions. I argue that a view of reduction inspired by Kemeny and Oppenheim's can be used to reduce levels of mechanisms.

Kemeny and Oppenheim's view is a kind of explanatory reduction, but it is considerably weaker than the Nagel/Schaffner model. Their motivating insight is that reduction is a type of scientific progress that involves an increase in the explanatory scope and systematization of theories. Their basic requirement is that the reducing theory must explain all of the observational data explained by the theory to be reduced. They do not require that the vocabularies of the reduced and reducing theories be connected via bridge laws. They require only that the reducing theory "fulfill the role" of the theory to be reduced.

This model of reduction has two distinct advantages. First, it can be interpreted as a relationship between levels of explanation and not necessarily between levels of theory. Second, it does not depend essentially on DN explanation for its primary motivation. Applied specifically to mechanistic explanation, a Kemeny-Oppenheim reduction requires reducing a higher-level phenomenon to a lower-level mechanism by demonstrating that the lower-level mechanism has an explanatory scope

\footnotetext{
${ }^{2}$ I do not use "terms" in the strong sense that carries commitments about the syntactic nature of explanation.
} 
at least as great as the phenomenon to be reduced. ${ }^{3}$ While mechanism and classical reduction are incompatible, there are good reasons for thinking that mechanism is at least compatible with the more general thesis of explanatory reduction. Chief among these reasons is the mechanists' assumption that mechanical systems are decomposable. ${ }^{4}$

Explaining a phenomenon mechanistically requires breaking the system into its parts and identifying the entities and activities that produce the phenomenon, as well as the ways in which those entities are arranged with respect to each other (spatially, temporally, or otherwise). Most accounts of mechanism emphasize the explanatory power furnished by the heuristic of decomposition. If a phenomenon can be explained mechanistically, we can infer that the system is decomposable.

Recall the thesis of explanatory reduction as applied to mechanisms: lower-level mechanisms can do all of the explanatory work performed by higher-level mechanisms, and perhaps more. If decomposing the system into its properties and parts yields an explanation of the phenomenon that contains no necessary reference to higher-level terms, then an explanatory reduction has been effected: decomposition involves explaining a higher-level phenomenon by appeal to lower levels. This mechanistic decomposition just is explanatory reduction. Decomposition, in turn, is essential to mechanistic explanation. Since mechanism involves a commitment to explaining a phenomenon in terms of the individual contributions of its parts, mechanists seem to be committed to explanatory reduction.

Indeed, several prominent mechanists have grown increasingly willing to characterize their projects as reductionistic. Bechtel now calls his view "mechanistic reduction":

\footnotetext{
${ }^{3}$ For a thorough treatment of this issue, see Theurer and Bickle (forthcoming 2013).

${ }^{4}$ See especially Bechtel and Richardson 1993.
} 
Insofar as a mechanistic explanation requires decomposing the mechanism into its working parts, and these are, in a well-delineated sense, at a lower level than the mechanism as a whole, mechanistic explanation is reductionistic. (Bechtel 2009, 20)

Craver (2007) echoes this point, noting that some philosophers and scientists use the term 'reduction' to refer to the explanation of a phenomenon by its mechanism.

But these views are not reductionistic in any strong sense. And that is to be expected. Historically, such observations have been a point of tension among mechanists. On one hand, mechanists aim to take scientific practice seriously. Wimsatt (1974) notes that many scientists see their search for mechanisms as reductionistic. But the aforementioned problems with classical reduction have generated a strong anti-reductionist consensus in philosophy. This has led most philosophers, including many mechanists, to distance themselves from most forms of reductionism. ${ }^{5}$ Many mechanists (Bechtel 2006, 2008; Bechtel and Abrahamsen 2005; Craver 2001, 2007) argue that their view is only weakly reductionistic: a phenomenon is reduced to the entities and activities that directly compose it but the reduction goes no further. On their view, complete explanations cannot be furnished by lower levels alone: complete explanations span multiple levels.

It appears that the New Mechanists are committed to two distinct claims that are prima facie incompatible:

(1) Mechanistic explanations are given in terms of the parts of the system, the properties of those parts, and the ways in which those parts are arranged.

(2) A complete mechanistic explanation of a systemic property requires appeal to higherlevel properties.

The first claim is basically a commitment to mechanism; the second, a commitment to antireductionism. Given that a weak version of explanatory reduction is built into mechanistic explana-

\footnotetext{
${ }^{5}$ Sarkar (2005) is a notable exception.
} 
tion and directly acknowledged by both Bechtel and Craver, New Mechanists must either reject one of these claims or find some way to render them compatible. But New Mechanists take both to be essential to their view.

Rejecting (1) amounts to a rejection of the centrality of decomposition as an explanatory tool in mechanistic explanation. Yet this is essentially what the New Mechanists do in embracing (2): they argue that complete explanations require appeal to multiple levels in a mechanistic hierarchy. But a key part of the appeal of mechanistic explanation is that decomposition is a powerful explanatory tool. ${ }^{6}$ The main alternative is the old DN model, to which no one is advocating an enthusiastic return.

But there is another option for the mechanist. She can reject (2): that is, deny that complete explanations require appeal to higher-level properties. From here, it is an easy transition to explanatory reductionism. This is precisely the strategy employed by Descartes, Boyle, and others, thus embracing explanatory reductionism. It is to this position that I now turn. Next, I show that the mechanical philosophy of Descartes and his contemporaries provides an alternative conception of mechanism that avoids the tension generated by claims (1) and (2) above.

\section{Old Mechanism}

Mechanism, in the seventeenth century sense of the term, was the dominant view in the natural philosophy of the time. It was, in many ways, a reaction to Aristotelian science. Most of the key figures in the science and philosophy of the day - Descartes, Hobbes, Huygens, Boyle, Harvey, Locke, Newton, Galileo, Borelli - can all, in some sense, be characterized as mechanists. But Old Mechanism was by no means a unified or monolithic movement. There were many substantive points of

\footnotetext{
${ }^{6}$ At least in neuroscience, the parts of a mechanism are typically at a lower level than the phenomenon to be explained. This may not be the case for mechanistic explanations in (e.g.) evolutionary biology or climate science, if those explanations are genuinely mechanistic.
} 
disagreement between all of these theorists. For the sake of time and simplicity, I focus on the views of Descartes and Boyle.

The central tenet that is shared by virtually all of the seventeenth century mechanists is that all natural phenomena could be explained by the mechanical behavior of the particles that make up a body - what the Old Mechanists called corpuscles. The behavior of a body is explained by the size, shape, and motion of the corpuscles that compose it. In the most radical form of Old Mechanism, explanations require nothing else. And at least for Descartes, those parts are generally small enough to elude the senses.

Descartes’ primary aim in developing his mechanistic worldview was to put biology, physiology, and even certain parts of psychology on a par with physical science: an aim that should sound strikingly similar to the reductionist project of the early to mid-twentieth century. The purpose of The Treatise on Man is, essentially, to explain all of human physiology in terms of the principles of Cartesian physics. Ideally, all of this could be explained in terms of the properties of fundamental particles. $^{7}$

Descartes himself says in the closing of The Treatise on Man, of the sweeping analysis of physiology presented to the reader:

I should like you to consider that these functions follow from the mere arrangement of the machine's organs every bit as naturally as the movements of a clock or other automaton follow from the arrangement of its counter-weights and wheels. In order to explain these functions, then, it is not necessary to conceive of this machine as having any vegetative or sensi-

\footnotetext{
${ }^{7}$ It must be noted that corpuscles are not fundamental in the sense of being indivisible. Corpuscularism is distinct from atomism: while they are both corpuscularists, neither Descartes nor Boyle are atomists (though some corpuscularists, such as Gassendi, were). Corpuscles are not indivisible, but they are fundamental in the sense that they form the ontological and explanatory basis for the entities they compose.
} 
tive soul or other principle of movement and life, apart from its blood and its spirits... $(1662 / 1998,202)$

The resources of Cartesian physics - or, perhaps more properly, mechanics - are in principle sufficient to explain the whole of the natural world. ${ }^{8}$

As Garber (2002) notes, the radical insight here is that there is no fundamental difference between the behavior of artificial and natural phenomena, and thus no difference in the explanations that such behaviors require. For Descartes, the only difference between artificial and natural phenomena concerns the size of the mechanisms at work. The mechanisms governing the behavior of natural phenomena are generally undetectable by the human eye, while the mechanisms underlying artificial phenomena are typically larger. But this, for Descartes, is not a substantive point about explanation. It is instead a result of constraints on what kinds of machines we can build: it is difficult to construct an artificial machine out of parts that cannot be observed or manipulated by human eyes and hands. But perhaps most radically, and as illustrated in the above passage, Descartes claims that even the human body is a kind of machine. As such, it should be explained by appeal to the same kinds of mechanical principles by which the behavior of machines is explained.

The New Mechanists have argued that their project is distinct from that of the Old Mechanists insofar as it is a reaction to an entirely different set of concerns about explanation. Indeed, it is important to situate these views in their proper historical context. Old Mechanism was primarily a response to Aristotelian science and to vitalism, as evidenced by the aforementioned passage in The Treatise on Man. In contrast, New Mechanism has functioned as an important corrective to the covering law models of explanation that dominated the philosophy of science in the twentieth century.

\footnotetext{
${ }^{8}$ It should be noted that Descartes' mechanical philosophy encompassed even certain psychological processes. He held that much of psychology - perception, emotion, even memory - could be explained mechanistically.
} 
These are important distinctions that should not be ignored. But these are differences of historical context that do not result in a fundamentally different conception of mechanistic explanation. And while historical and contextual factors have certainly shaped both views, the view of explanation that we find in both New and Old Mechanism is perhaps more similar than the New Mechanists have typically recognized. The major difference between the two views is that New Mechanism is supposedly less reductionistic. But as I have demonstrated in the previous section, this relies on two claims about New Mechanism that cannot be endorsed simultaneously without risking incoherence.

\section{Mechanism, Corpuscularism, and Mechanistic Reduction}

Old Mechanism can be separated into two distinct claims, which Glennan (1992) terms corpuscularism and mechanism. Corpuscularism is a thesis about the micro-structure of matter. Corpuscles are the fundamental constituents of matter; the ultimate furniture of the world. The corpuscular hypothesis is that all natural phenomena arise from the motions of these corpuscles, which come in different shapes and sizes. Some of the greatest scientific achievements of corpuscularism - and one of the clearest commitments to it - can perhaps be attributed to Boyle.

A dominant theme in Boyle's work is the idea that chemical reactions are merely the reshuffling of particles, and that all properties of chemistry are the products of the motions of corpuscles. These corpuscles form larger particles, which constitute the entities that are the proper subject matter of chemistry.

The second claim of Old Mechanism is what Glennan simply calls mechanism. This is a methodological approach toward understanding nature. The natural world is to be understood as a kind of machine, and natural phenomena are to be explained by identifying the parts of the machine that contribute to the production of the phenomenon to be explained. 
On Glennan's view, the logical relationship between mechanism and corpuscularism is one of one-way implication: corpuscularism entails mechanism, but not vice versa. Both claims, taken together, constitute the view of the Old Mechanists - what Glennan calls strict mechanism:

Strict Mechanism is the view that all legitimate scientific explanations are to be given in terms of "strictly mechanical principles," where strictly mechanical principles are taken to be principles of action by contact, either by collision or by more extended pushes. (Glennan 1992, 16)

Glennan, along with nearly all of the New Mechanists (as we've already seen), embraces Mechanism but not corpuscularism, thereby rejecting Strict Mechanism. For Glennan and the rest of the New Mechanists, Strict Mechanism is untenable for two reasons. First, Glennan argues that corpuscularism has been demonstrated empirically to be false. The corpuscular hypothesis would have precluded mechanists from embracing such "occult" forces as gravity, electromagnetism, and nuclear forces. Of course, such forces have proven to be indispensable to our current understanding of physics.

But the problem is not merely that corpuscularism is empirically inadequate. After all, corpuscularism isn't a strictly empirical thesis. It has a philosophical component as well: namely, that there are fundamental constituents of matter in terms of which everything else can be explained. Whether or not we're right about what those constituents are is strictly an empirical matter, but the commitment to some fundamental particles is a commitment with both an empirical and a philosophical component. Rejecting corpuscularism on the grounds that it is empirically inadequate won't be sufficient reason for us to discount the thesis entirely. Even if it is empirically inadequate as it stands, there still may be something right about the philosophical component of corpuscularism. But Glennan's difficulty with corpuscularism is philosophical as well:

The Corpuscular theory may easily be criticized for an excessive commitment to reduction. The view advocated by Descartes and others was that the truly scientific explanations must be 
given in terms of the lowest level organization of nature, that is in terms of the size, shape and motion of corpuscles. In the language of contemporary theories of reduction, the Corpuscularist was an eliminative reductionist. This overcommitment to reduction may explain the relative lack of progress in the higher-level sciences. (1992, 15, emphasis mine)

Indeed, the basic view of mechanism plus corpuscularism is strikingly similar to the project of explanatory reductionism.

An ideal explanation for Descartes (and many of his contemporaries) would offer a series of pictures in increasingly finer detail, exhibiting a nested series of mechanisms capable of explaining all that occurs in the organism. This idea strongly resembles the view of interlevel relationships advocated by many contemporary reductionists, as vividly represented by Churchland and Sejnowski (1992). On their view, downward-looking explanation involves starting with the central nervous system and investigating progressively lower levels. This general motif is strikingly similar to Descartes' view in The Treatise on Man. In Des Chene's analysis of Descartes' project in The Treatise on Man, he notes:

Descartes shows how cycles of bodily activity—the pulse, the wasting and replenishment of the blood, the consumption and the acquisition of food—can be explained: the body is to be analyzed into systems of mechanisms, and each mechanism into simpler mechanisms, until we arrive at mechanisms whose capacities can be understood in terms of the modes and derived properties of extended things. (Des Chene 2001, 154).

For Descartes, an ideal explanation of human physiological processes requires investigating progressively lower levels in a mechanistic hierarchy, until we reach a mechanism that can be described in purely physical terms. Garland Allen makes a similar point:

Mechanists have tended to hold to some kind of reductionist strategy, that is, the belief that to understand higher level processes it is necessary to investigate them at lower levels of or- 
ganization....In this formulation Mechanistic materialism is downward-looking, proceeding from higher to lower levels of organization in the search for the most general and basic explanation. (Allen 2005, 266)

Descartes' mechanistic worldview captures exactly the main thesis of explanatory reductionism: that lower-level mechanisms can explain higher-level phenomena without necessary appeal to higherlevel terms, as the New Mechanists have argued. Garber underscores this point:

[Descartes'] point in establishing a correspondence between the artificial machine and the natural body was that we can now explain everything that happens in the natural body in exactly the same way in which we explain what happens in the artificial machine. That is, we explain things in the natural world as if they had been made by a mechanic who has only parts of different sizes, shapes, and motions to work with when producing his effects. (Garber 2002, 198)

A mechanical system is a collection of parts arranged in particular ways, as determined by the size, shape, and motions of the parts, as well as their interactions. That is all that is required for an explanation of the machine's behavior. Explanation requires no appeal to higher-level properties or to the intrinsic purposes of the system. ${ }^{9}$ This fundamental commitment is shared by both the Old Mechanists and the reductionists.

But this is precisely what Glennan and the rest of the New Mechanists reject. Glennan's position - mechanism without corpuscularism - is what he calls "methodological mechanism":

\footnotetext{
${ }^{9}$ One might argue that explanation of systemic behavior requires first seeing the system as doing something: as having a function or purpose. Functions are certainly necessary for identifying the explanandum phenomenon. But there is no need for the reductionist to dispute this because the function itself is not part of the explanans. Interestingly, Garber makes a similar point about Descartes' own view: "The purpose of a machine may determine what behavior it is that we are trying to produce, but it does not enter into the way in which the behavior itself is produced" $(2002,198)$.
} 
Methodological Mechanism is a weaker view which amounts to the adoption of a particular metaphor, the machine metaphor, in which the world is seen as being like a machine. The methodological implication of this metaphor is the suggestion that natural phenomena should be explained in roughly the same way as the behavior of machines is explained. (Glennan 1992, 16)

Adopting this position requires some way of guarding against the excessive reductionism of corpuscularism, which is exactly what the New Mechanists' attempts at articulating a workable account of mechanism compatible with anti-reductionism are designed to do.

But the failure to dissociate mechanism and reductionism fully suggests that the connection between mechanism and corpuscularism (or reductionism) is tighter than Glennan's analysis suggests. In fact, I have argued that explanatory reduction is built into the very conception of mechanism that the New Mechanists have articulated. But even if there is no strict logical entailment from mechanism to corpuscularism, such that methodological mechanism is an available position in the logical space, the problems that I have raised here suggest that dissociating mechanism and reductionism in practice is more difficult than the New Mechanists have taken it to be. Finally, it must be emphasized that the intimate historical connection between reductionism and mechanism provides a resource that reductionists should embrace in defense of their view, rather than continue the fruitless attempt to save a law-based model of reduction. 


\section{REFERENCES}

Allen, Garland E. 2005. "Mechanism, Vitalism and Organicism in Late Nineteenth and Twentieth Century Biology: The Importance of Historical Context." Studies in the History and Philosophy of the Biological and Biomedical Sciences 36: 261-283.

Bechtel, William. 2006. Discovering Cell Mechanisms: The Creation of Modern Cell Biology. New York: Cambridge University Press.

—. 2008. Mental Mechanisms: Philosophical Perspectives on Cognitive Neuroscience. London: Routledge.

- 2009. "Molecules, Systems, and Behavior: Another View of Memory Consolidation." In J. Bickle (ed.), Oxford Handbook of Philosophy and Neuroscience. Oxford: Oxford University Press.

Bechtel, William and Adele Abrahamsen. 2005. "Explanation: A Mechanist Alternative." Studies in the History and Philosophy of Biological and Biomedical Sciences 36: 421-441.

Bechtel, William and Robert C. Richardson. 1993. Discovering Complexity: Decomposition and Localization As Strategies in Scientific Research. Princeton, NJ: Princeton University Press.

Bickle, John. 1998. Psychoneural Reduction: The New Wave. Cambridge, Massachusetts: The MIT Press.

- 2003. Philosophy of Neuroscience: A Ruthlessly Reductive Approach. Dordrecht, Holland: Kluwer Academic Publishers.

- 2006. "Reducing Mind to Molecular Pathways: Explicating the Reductionism Implicit in Current Cellular and Molecular Neuroscience." Synthese 151: 411-434.

Churchland, Patricia S. 1986. Neurophilosophy: Toward a Unified Science of the Mind/Brain. Cambridge, Massachusetts: The MIT Press.

Churchland, Patricia S. and Terrence J. Sejnowski. 1992. The Computational Brain. Cambridge, MA: MIT Press.

Craver, Carl F. 2001. "Role Functions, Mechanisms, and Hierarchy." Philosophy of Science 68: 53-74.

- 2007. Explaining the Brain: Mechanisms and the Mosaic Unity of Neuroscience. New York: Oxford University Press.

Descartes, René. 1998. The World and Other Writings, ed. Stephen Gaukroger. Cambridge: Cambridge University Press.

Descartes, René. 1662/1998. The Treatise on Man. In Descartes (1998).

Des Chene, Dennis. 2001. Spirits and Clocks: Machine \& Organism in Descartes. Ithaca, NY: Cornell University Press. 
Fodor, Jerry. 1974. "Special Sciences (Or: The Disunity of Science As a Working Hypothesis)." Synthese 28: 97-115.

Garber, Daniel. 2002. "Descartes, Mechanics, and the Mechanical Philosophy." Midwest Studies in Philosophy 26: 185-204.

Glennan, Stuart S. 1992. "Mechanisms, Models, and Causation.” PhD diss., University of Chicago.

—. 1996. "Mechanisms and the Nature of Causation." Erkenntnis 44: 49-71.

—. 2002. "Rethinking Mechanistic Explanation." Philosopby of Science (Suppl.) 69: S342-53.

Kemeny, John G. and Paul Oppenheim. 1956. “On Reduction.” Philosophical Studies 7: 619.

Machamer, Peter K., Lindley Darden, and Carl F. Craver. 2000. “Thinking About Mechanisms.” Philosophy of Science 57: 1-25.

Putnam, Hilary. 1975a. “The Nature of Mental States.” In Putnam (1975b).

—. 1975b. Mind, Language and Reality: Philosophical Papers, Volume 2. New York: Cambridge University Press.

Nagel, Ernest. 1961. The Structure of Science: Problems in the Logic of Scientific Explanation. Indianapolis, IN: Hackett Publishing Company.

Rosenberg, Alex. 1997. "Computing the Embryo: Reduction Redux.” Biology and Philosophy 12: 445470.

- 2006. Darwinian Reductionism: Or, How to Stop Worrying and Love Molecular Biology. Chicago: The University of Chicago Press.

Sarkar, Sahotra. 2005. Molecular Models of Life: Philosophical Papers on Molecular Biology. Cambridge, MA: MIT Press.

Schaffner, Kenneth F. 1967. “Approaches to Reduction.” Philosophy of Science 36: 280-290.

- 1993. Discovery and Explanation in Biology and Medicine. Chicago: University of Chicago Press.

Theurer, Kari L. and John Bickle. Forthcoming 2013. “What's Old Is New Again: KemenyOppenheim Reduction in Current Molecular Neuroscience.” Philosophia Scientiae 17/2 (special issue on "The mind-body problem in cognitive neuroscience").

Wimsatt, William C. 1974. "Reductive Explanation: A Functional Account." Proceedings of the Biennial Meeting of the Philosophy of Science Association 671-710.

Woodward, James. 2003. Making Things Happen. New York: Oxford University Press. 